\title{
Optimization of processing parameters for the preparation of phytosterol microemulsions by the solvent displacement method
}

\begin{abstract}
The purpose of this study was to optimize the parameters involved in the production of watersoluble phytosterol microemulsions for use in the food industry. In this study, response surface methodology (RSM) was employed to model and optimize four of the processing parameters, namely, the number of cycles of high-pressure homogenization (1-9 cycles), the pressure used for high-pressure homogenization (100-500 bar), the evaporation temperature $\left(30-70{ }^{\circ} \mathrm{C}\right)$, and the concentration ratio of microemulsions (1-5). All responses-particle size (PS), polydispersity index (PDI), and percent ethanol residual (\%ER) - were well fit by a reduced cubic model obtained by multiple regression after manual elimination. The coefficient of determination (R2) and absolute average deviation (AAD) value for PS, PDI, and \%ER were 0.9628 and $0.5398 \%, 0.9953$ and $0.7077 \%$, and 0.9989 and $1.0457 \%$, respectively. The optimized processing parameters were 4.88 (approximately 5) homogenization cycles, homogenization pressure of 400 bar, evaporation temperature of $44.5^{\circ} \mathrm{C}$, and concentration ratio of microemulsions of 2.34 cycles (approximately 2 cycles) of high-pressure homogenization. The corresponding responses for the optimized preparation condition were a minimal particle size of $328 \mathrm{~nm}$, minimal polydispersity index of 0.159 , and $<0.1 \%$ of ethanol residual. The chi-square test verified the model, whereby the experimental values of PS, PDI, and \%ER agreed with the predicted values at a 0.05 level of significance.
\end{abstract}

Keyword: Phytosterols; Microemulsions; Solvent displacement method; Response surface methodology; High-pressure homogenization 